REVIEW

\title{
Component coding and the neurointerventionalist: a tale with an end
}

\author{
Joshua A Hirsch, ${ }^{1}$ William D Donovan, ${ }^{2}$ Thabele M Leslie-Mazwi, ${ }^{1}$ Greg N Nicola, ${ }^{3}$ \\ Laxmaiah Manchikanti, ${ }^{4}$ Ezequiel Silva III $^{5}$
}

${ }^{1}$ Neuroendovascular Program, Massachusetts General Hospital, Harvard Medical School, Boston, Massachusetts, USA

${ }^{2}$ Norwich Diagnostic Imaging Associates, Norwich,

Connecticut, USA

${ }^{3}$ Department of Radiology,

Hackensack University Medical

Center, Hackensack,

New Jersey, USA

${ }^{4}$ Pain Management Center of Paducah, Paducah, Kentucky, USA

${ }^{5}$ South Texas Radiology Group, San Antonio, Texas, USA

\section{Correspondence to} Dr J A Hirsch,

Neuroendovascular Program, Massachusetts General Hospital, Harvard Medical School, Boston, MA 02114, USA; Hirsch@snisonline.org

Received 26 November 2012 Revised 26 November 2012 Accepted 26 November 2012 Published Online First 19 December 2012

To cite: Hirsch JA, Donovan WD, Leslie-Mazwi TM, et al. J Neurolntervent Surg 2013;5:615-619.

\section{ABSTRACT}

Component coding is the method Neurolnterventionalists have used for the past 20 years to bill procedural care. The term refers to separate billing for each discrete aspect of a surgical or interventional procedure, and has typically allowed billing the procedural activity, such as catheterization of vessels, separately from the diagnostic evaluation of radiographic images. This work is captured by supervision and interpretation codes. Benefits of component coding will be reviewed in this article. The American Medical Association/Specialty Society Relative Value Scale Update Committee has been filtering for codes that are frequently reported together.

Neurolnterventional procedures are going to be caught in this filter as our codes are often reported simultaneously as for example routinely occurs when procedural codes are coupled to those for supervision and interpretation. Unfortunately, history has shown that when bundled codes have been reviewed at the RUC, there has been a trend to lower overall RVU value for the combined service compared with the sum of the values of the separate services.

\section{INTRODUCTION}

Component coding refers to separate billing for each discrete aspect of a surgical or interventional procedure, and has been the de facto method of documenting the panorama of neurointerventional (NI) care for 20 years. ${ }^{1}$ It has usually entailed separate billing of the surgical/procedural activity, such as catheterization of vessels, from the diagnostic evaluation of radiographic images (supervision and interpretation code (or S\&I)) performed for an NI service. For most practicing NeuroInterventionalists, component coding represents the only system we have ever used for billing procedural care. NI specialists might be surprised by the relative youth of component coding, and the peril that this now established billing system currently faces.

\section{REAL LIFE SCENARIO}

In April 2012, the American Society of Neuroradiology and the American College of Radiology (ACR), as well as a litany of other societies including the Society of Interventional Radiology and the American Association of Neurological Surgeons, presented a new set of bundled carotid angiography code services to the RUC (the American Medical Association/Specialty Society Relative Value Scale Update Committee) for recommendation of relative work value units (RVU). The cervicocerebral angiography codes had been identified as 'potentially misvalued' by the RUC through their '75\% reported together' screen.

The societies argued that the multiple individual component codes were originally valued independently, and on their own merits; therefore, there were no inherent efficiencies when the services were performed together. Supported by survey data from practicing physicians, the RUC agreed that when a new code bundle involves a single procedure and its S\&I code, the new code RVU value should be the sum of the two 'old' code values. The RUC forwarded these recommendations to the Centers for Medicare and Medicaid Services (CMS).

CMS did not agree, categorically stating that "We believe efficiencies are gained when services are bundled". 2 Accordingly, CMS lowered the RUC's recommended values for the new cervicocarotid code set in the November 2012 Final Rule-unilaterally rejecting the notion that work efficiencies did not exist between procedural codes and S\&I codes. The reductions for diagnostic cervicocerebral angiography are large. For example, a four vessel diagnostic cerebral angiogram now suffers a $44 \%$ reduction in payment compared with the prior conventional component coding structure.

\section{BRIEF HISTORICAL BACKGROUND}

In December 1989, President George H W Bush signed the Omnibus Budget Reconciliation Act of 1989 into law. This act established a Resource Based Relative Value System (RBRVS) as the basis for Medicare physician reimbursement, which took effect on January 1, 1992.

Prior to 1992, interventional services were reported using two different methods: complete procedure coding or component coding. At that time, the vast majority of percutaneous vascular interventional procedures were performed and coded by radiologists (with the exception of cardiology procedures). Thus professional radiology organizations had a central role in developing a working plan for interventional and NI coding. ${ }^{3}$

Having two separate coding conventions led to significant variability in the reporting of procedures. Furthermore, different insurance companiesincluding regional Medicare contractors-would reimburse variably and sometimes uniquely for the services performed. Some would pay as if only a single procedure had been performed whereas others reimbursed separately for the different components of the interventional procedure. This was confusing to providers as well as payers. 
Prior to and during the 1980 s, the ACR crafted its own Radiology RVS through tremendous research, survey, and analysis efforts. ${ }^{4}$ Based on the strength of the proposed system, in 1989 the Health Care Financing Administration (HCFA, now CMS) agreed to adopt the Radiology RVS essentially wholesale into its prospective RBRVS. The ACR's RVS focused on diagnostic procedure valuation but the ACR did recommend the elimination of complete procedure codes in favor of a component coding system. However, HCFA could not make such a large change to the coding system as only the American Medical Association's (AMA) Current Procedural Terminology (CPT) Editorial Panel (EP) could delete and add CPT codes. Therefore, the ACR assigned relative values to the interventional radiology codes which existed in 1989.

Between 1989 and 1992, the Society of Cardiovascular and Interventional Radiology (SCVIR), now the Society of Interventional Radiology, proposed to the CPT EP a system in which both complete and component coding methods would be used to describe interventional procedures. The AMA rejected this proposal as too complex.

In 1991, SCVIR and ACR developed a second, simpler proposal establishing component coding as the basis for reporting interventional radiology procedures. This proposal was accepted by both HCFA and the AMA. This was a watershed moment in radiologist reimbursement, thanks to the prolonged efforts of SCVIR and ACR physician volunteers and staff; it heralded the 20 year run of the component coding classification which has been the basis of describing most NI procedures-until recently.

\section{ADVANTAGES OF COMPONENT CODING}

There are multiple benefits of component coding:

1. It allows separate billing of surgical/interventional codes and S\&I codes-particularly important when different physicians carry out these services on the same patient.

2. The detail built into the system allows separate reporting for procedures performed in different vascular beds (eg, carotid vs vertebral arteries), which was generally not a part of the prior reporting conventions.

3. It allows for differential valuation for greater selectivity/ complexity (eg, catheterizing the internal carotid artery is reimbursed higher than the common carotid artery).

4. It is substantially more comprehensive, leaving few clinical services undescribed, and therefore fewer clinical scenarios billed as an 'unlisted procedure,' subject to absent or irregular coverage by insurers.

5. Component coding facilitates adaptation to clinical development and innovations: by allowing more efficient and discrete tracking of work performed within a department, resource planning and allocation is more refined. New technologies can integrated more rapidly into the existing coding structure, rather than facing the delays of new code creation.

6. The increased granularity of the procedural tracking has important implications for research: research studies can focus on specific services by means of more specific CPT codes, and research grants can be more effectively sought and awarded when the stakeholders understand what is being proposed to a more detailed degree.

Throughout both the adolescence of the component coding structure, and whenever new technology has led to new CPT codes in the years since, the RUC has meticulously reviewed and valued each component service on its own merit. Thus the reimbursement convention that has existed for two decades has assumed that payment for each component service, or any combination of components, was appropriate for the service performed.

These aspects of the component coding system-that each code's value is inherent, and that multiple component codes may be used for a single patient interventional visit-has been of great value to the practice of radiology and other procedural specialties. However, of late, they have also become the nexus of unflagging criticism from health care pundits and CMS policymakers. ${ }^{1}$

\section{THE TIDE TURNS}

In this era of diminishing Federal resources, a growing number of voices have raised concerns that component coding has led to inappropriate reimbursement. MedPAC (the Medicare Payment Advisory Commission) and the Government Accountability Office, among others, have reiterated that the current health care system is financially unsustainable. ${ }^{5} 6$ They have included radiologist reimbursement in general-and component coding in particular-as harvest ready, low hanging fruit to redistribute payments to other physicians.

A little more background: to preserve the rigor of the RUC database, a '5 year review' (5YR) process was statutorily mandated at the inception of the RBRVS, and has taken place every 5 years through the RUC. Designed to maintain relativity in the face of changing practice and evolving technology, the first $5 \mathrm{YR}$ took place in 1995. By 2005, critics such as MedPAC charged that the RUC was not reviewing codes often enough or critically enough. ${ }^{5}$ CMS and the RUC agreed. Thus, following the third $5 \mathrm{YR}$, an ongoing review process was instituted. ${ }^{7}$ Originally termed 'the rolling 5YR,' it was immortalized in 2009 as the Relativity Assessment Workgroup (RAW), a standing subcommittee of the RUC, with ongoing recommendations. ${ }^{8}$

RAW's mandate from CMS has been to review 'potentially misvalued services'. CMS directed RAW toward several different categories of services by means of 'screens' or 'filters.' Of particular interest to neurointerventionalists is the screen of 'codes frequently performed together'. When CMS and RAW developed the screen for 'codes frequently performed together,' a joint CPT/RUC workgroup was formed to consider the complex coding issues inherent to codes 'reported together'. The hope of the radiology community was that any potential resource efficiencies would be contextualized, given (1) the historic rationale for and development of component coding and (2) the potentially devastating effect that may befall a few procedural specialties.

The workgroup's report to the RUC and CPT EP 'called for CPT coding change proposals to collapse code pairings into fewer bundled services'. The reported together screen started at $95 \%$ or more reported together; in 2010, the threshold was lowered to $75 \%$ or more reported together. ${ }^{9}{ }^{10}$ Code pairs meeting this criterion were sent to the joint CPT/RUC work group. In nearly all cases, such code pairs or families have been reformulated as bundled codes. And in most cases, a new bundled code is valued less at the RUC and by CMS than the sum of the values of the previous codes. ${ }^{11}$ Given the prevalence with which NI procedures are reported with S\&I codes, and only somewhat less commonly with each other, numerous NI procedures have been identified for examination-and have been or may ultimately be reimbursed less than they are currently.

The family of cervicocarotid angiography codes is the most important group of services to NI radiology that has been reformulated through this process to date (see box 1 ). 


\section{Box 1 An example of the code bundling process: cervicocarotid angiography}

Numerous code pairs from the family of diagnostic carotid artery procedures were identified in the 'potentially misvalued' screen of 'codes performed together' more than $75 \%$ of the time

- Code pairs were identified by Centers for Medicare and Medicaid Services (CMS) data analysis in February 2010.

- Reviewed at the Relativity Assessment Workgroup (RAW) meeting in April 2010. The RAW/RUC (American Medical Association/ Specialty Society Relative Value Scale Update Committee) agreed to schedule this family for Current Procedural Terminology (CPT)/ RUC review almost 2 years hence-because of the overall workload (including many other code families) being imposed on a relatively small number of societies, especially radiology.

- Large number of societies (American Society of Neuroradiology, Society of Interventional Radiology, American College of Radiology, neurosurgical, cardiology, and vascular surgery organizations) worked together to revise the current code structure, presented to CPT in February 2012.

- New codes all include the radiological supervision and interpretation (S\&I) code, fluoroscopic guidance, roadmapping, and initial vascular access. Like the current code structure, the procedure codes are progressive- the service reflecting the highest order branch vessel is coded, and also includes all work leading up to that level.

- Societies surveyed their membership, analyzed the data collectively, and presented their recommendations to the RUC in April 2012.

- Example from the RUC meeting: Complete right internal carotid artery angiography.

$\triangleright$ Societies recommended value of 7.55 .

$\triangleright$ RUC agreed, forwarding that recommendation to CMS.

$\triangleright$ Value diminished by CMS in Final Rule to 6.50.

- New code values were published in CMS Final Rule in November 2012, taking effect 01/01/2013**.

Examples:

1. Right internal carotid diagnostic angiography

Previous valuation: Catheterization of a third order brachiocephalic arterial branch (36 217) plus radiologic S\&I (75 665)=6.29+1.31 relative work value units $(\mathrm{RVU})=7.60 \mathrm{RVU}$.

New valuation (beginning 01/01/2013): Unilateral internal carotid artery angiography, includes catheter placement, arch and common carotid artery angiography, S\&I (36 224): 6.50 RVU.

Bundled code value discounted $14 \%$ from prior component code valuation.

2. Bilateral vertebral arteriography

Previous valuation: Right vertebral catheterization (third order) (36 217), left vertebral artery catheterization (second order) (36 216$)^{*}$, vertebral arteriography S\&I $(75685 \times 2)=6.29+2.64 *+1.31+1.31=11.55$ RVU.

New valuation: There are now separate vertebral angiography codes for non-selective (ie, injecting the subclavian artery for vertebral artery evaluation (36 225)) and selective catheterization and angiography of the vertebral artery itself (36 226). The new codes no longer refer to first, second, or third order anatomy.

Assuming selective bilateral vertebral artery catheterization: $36226+36226-50$ (bilateral modifier) $=6.50+3.25^{*}=9.75$.

Bundled code valuation decrease of $15 \%$.

Note: Intracranial catheterization and angiography reported by means of an add-on code 36228 (4.25 RVU), reporting limited to twice per side.

Any endovascular interventions performed following these diagnostic procedures are still reported separately, except that carotid stenting codes include preliminary catheterization and diagnostic arteriography.

Ultrasound guidance for initial vascular access (76 937) still reported separately.

Three-dimensional rendering $(76376,76377)$ still reported separately.

Upper extremity arteriography codes may still be reported separately.

*Most payers, including Medicare, discount the second procedure payment for a bilateral procedure.

** CMS reduced the value of CPT 36224 stating that the 25 th percentile survey value appropriately captures the work of the service, 'particularly efficiencies when two services are bundled together'. The society recommended reference services were ignored and no alternative reference codes provided by CMS (Reference: 2013 MPFS Final Rule, page 508)

\section{WHY?}

There are both rational and emotional explanations for the targeting of radiology and interventional codes by the payers and policymakers. $^{12}$

The genesis of the 'codes reported together' screen was the concept that services that are reported together an overwhelming percentage of the time are not truly separate services, but different parts of the same procedure. This logic is difficult to refute. By bundling two or more such services into a single redefined entity, there is a presumed simplification of the coding structure.

As it turns out, when bundled codes have been reviewed at the RUC, there has been a steady trend for diminution of overall RVU value for the combined service compared with the sum of the values of the separate services. This is exemplified by the story of inferior vena cava filter placement (see box 2), and on the diagnostic side, the combined codes for CT of the abdomen and pelvis. ${ }^{11}$ This trend has been seen by policymakers as a justification for this process. Policymakers would point to the reduced value of the combined code as evidence of overlap in work between the previously separately reported codes-that 'efficiencies' have been captured. (Paradoxically, code bundling has not reduced the number of CPT codes, but increased them. Many of the new code families are also complex and numerous; and many of the individual component codes have been maintained in the code set to cover unusual 
IVC filter placement

Inferior vena cava (IVC) filter placement is a commonly performed procedure in hospitalized patients. Neurologically impaired patients are often the recipients of such devices. IVC filter utilization has increased as a method to prevent life threatening pulmonary emboli associated with deep venous thrombosis, particularly in patients with a contraindication to anticoagulation, a frequent circumstance in neurointerventional patients. ${ }^{13} 14$

Traditional coding (pre-2010)*

36010 (introduction of catheter, superior vena cava or IVC); 37620 (interruption of IVC); and 75940 (filter placement supervision and interpretation (S\&I)): combined relative work value units (RVU) value $2.43++11.57+0.54^{* *}=12.11$

- Code pairs 36 010/37 620 and 37 620/75 940 were identified by the joint American Medical Association/Specialty Society Relative Value Scale Update Committee (RUC)/Current Procedural Terminology (CPT) workgroup in the 'codes performed together' screen in 2010.

- American College of Radiology, Society of Interventional Radiology, and the Society for Vascular Surgery agreed to send the family of codes to CPT for revision, bundled code creation.

- CPT approved new code set in February 2011; CPT deleted codes 37620 and 75490 from the CPT guidebook. 36010 is maintained as standalone codes.

- The same societies presented their survey results and recommendations to the RUC in April 2011. The intra-service times returned in the surveys could not justify maintaining the existing values.

RUC recommendations to Centers for Medicare and Medicaid Services (CMS)

- 37191 (IVC filter placement, including imaging guidance, roadmapping, S\&l): 4.71 RVU

- 37192 (IVC filter repositioning, including imaging guidance, roadmapping, S\&l): $8.00 \mathrm{RVU}$

- 37192 (IVC filter retrieval/removal, including imaging guidance, roadmapping, S\&I): 8.00 RVU CMS final determinations

- 37 191: accepted RUC recommendation, $4.71 \mathrm{RVU}^{* * *}$

- 37 192: downvalued the RUC recommendation. 7.35 RVU

- 37 193: downvalued the RUC recommendation, 7.35 RVU

*Additional procedures were sometimes billed as well for this procedure. The four codes listed were for the most common coding scenario.

**75 940 (S\&I) was carrier priced from 2007-2011. 0.54 RVU was the CMS value prior to that time.

${ }^{* * *} A 61 \%$ reduction in payment from prior coding.

cases/scenarios. Nonetheless, more unreportable procedures have ensued, such as the new renal angiography codes where a right sided subselective and left sided superselective catheterization is not reportable in the new bundled scheme for diagnostic renal angiography.)

The less rational attractiveness of the code bundling movement has to do with a negative perception of the fee for service structure of the American health care system, and the specialties that have benefited most from this system-including radiologists and other proceduralists. ${ }^{15}$ The average primary care physician is frequently limited to reporting a single evaluation and management (E\&M) code per patient visit. Such providers shudder at the thought that neurointerventionalists may report five, 10 , or 20 codes for a single patient encounter. There is a perception that primary care providers are underpaid, and medical students are turning away from those fields. Some go on to say that this is a 'ticking time bomb,' or evidence of a failing American medical system. ${ }^{16}$

The concept that the fee for service system rewards those who can report more codes, regardless of the necessity for or the quality of the care provided, is reinforced, detractors say, by the RUC itself. The RUC has a plurality of specialists, and some assert that it is unwilling to address the pay gap in a meaningful way. The AMA and RUC itself have maintained a staunch defense of their traditions, and of their fair and objective approach to the valuation of medical and surgical procedures. ${ }^{17}$ However, while CMS accepted greater than $90 \%$ of the RUC's RVU recommendations until a few years ago, it has of late more frequently adjusted the RUC's valuations in its Final Rules- signaling to some an attempt to show more backbone to its detractors. In fact, the RUC recently approved two additional seats on the panel for primary care specialists.

\section{MULTIPLE PROCEDURE PAYMENT REDUCTION POLICY}

Another tactic used by CMS to trim health care costs at the expense of radiologists and proceduralists is the progressive expansion of the Multiple Procedure Payment Reduction policy (MPPR). Rather than wait for RAW, CPT and RUC to address the hundreds of codes on its agendas every year-only some of which lead to bundling and 'captured efficiencies'-CMS has sought through its regulatory powers to unilaterally discount 'second and subsequent' procedures on its own.

In 2006, CMS begin discounting the technical reimbursement for second and subsequent imaging and surgical services provided to Medicare patients by the same physician on the same day-initially at $25 \%$, subsequently raised by Congress to $50 \%$.

Other MPPR targets on the technical side have included nuclear medicine codes (especially prior to recent code bundling), physical therapy services, and more recently cardiovascular and ophthalmology codes. Surgical services have had an MPPR in place for 90 day global codes since 1991, largely the basis for the recent MPPR expansion.

In 2009, the General Accounting Office launched a direct broadside against the component coding structure in a report to Congress titled, 'Fees could better reflect efficiencies when services are provided together'. ${ }^{6}$ The basic argument was that physician reimbursement should also be discounted when services were provided together, just as the technical payments had been. 
CMS has taken up the challenge of finding efficiencies by introducing a professional MPPR on advanced imaging services in 2012, and expanding it to include all members of the same radiology practice for $2013 .^{2}$

While NI practitioners might assume that the expanded MPPR might protect against future code bundling, in that it would prima facie capture implied efficiencies of single encounter NI service, this has not been the case. ${ }^{18}$ Although the professional MPPR has reduced reimbursements on a case by case basis more than CMS has ever been able to logically justify, it has not been considered an adequate rationale for removing services from the 'codes frequently used together' RUC screens. The RUC asserts that it does not address or respond to CMS policy, and will therefore continue its work to seek efficiency through bundling, where appropriate.

\section{FUTURE CONCERNS}

The Patient Protection and Affordable Care Act (PPACA, or 'Obamacare') empowers the Secretary of the Department of Health and Human Services, of which CMS is one department, to 'periodically identify services as being potentially misvalued' and states that the secretary may consolidate individual codes into bundled codes for payment'-for example, potentially bypassing the RUC-CPT process entirely. ${ }^{19} 2021$

Another provision of PPACA due to begin its work in 2014 is the Independent Payment Advisory Board (IPAB). The President, in consultation with congressional leaders, would have the authority to name 15 experts to serve on this commission, whose purpose would be to seek cost savings from the Medicare process. They would have almost unchallengeable authority over a broad spectrum of reimbursement issues: from resetting RVU values for individual procedures, to amending practice expense inputs, to setting new payment initiatives. This institution could therefore further marginalize the RUC. The AMA and virtually every medical specialty society has gone on record opposing the IPAB, as it would potentially take medical and surgical procedure valuation another step further away from physician input.

CMS has also recently indicated that they have 'entered into two contracts to assist us in validating RVUs of potentially misvalued codes'. ${ }^{2}$ No further details have been provided.

\section{COGNITIVE DISSONANCE}

Component coding was specifically designed as granular and highly detailed, and was instituted with the imprimatur of the AMA and HCFA. Reporting certain codes together was innate to the system. The component codes were properly and explicitly valued as discrete services when they were created, and have been reviewed and reinforced regularly since. ${ }^{7}$ Now the powers that be have decided that component coding is inefficient, and may be contributing to inappropriate reimbursement. Thus numerous component codes have been identified on RAW screens as 'potentially misvalued,' with specialty societies forced to work through a bundling process at CPT, and then a survey process and presentation to the RUC for new valuation. ${ }^{12}$

\section{CONCLUSION}

CMS is under tremendous pressure to redistribute payments to primary care physicians over specialists and, where possible, lower health care costs. Over the past several years, the RUC has been challenged for favoring specialists over primary care providers, and for a non-aggressive approach toward review of previously valued codes. The RUC, AMA, and many specialty societies have become concerned about maintaining the RUC's critical role in determining the value of physician services. The authors of this short review are convinced that the working of the RUC remains the most just way of maintaining relativity across the spectrum of care. Recent initiatives by CMS and provisions of PPACA may further marginalize the influence of the RUC, and by extension of physicians in general.

As a matter of legislative policy, as well as precedent, the RUC has increasingly focused on identifying 'potentially misvalued services,' many in the interventional area. The application and results have been far from uniform. The 'frequently reported together' screen used by the RAW and thus the RUC stands as a clear challenge to component coding. Many NI procedures have been or are at significant risk of being bundled. If past history is any guide, such bundling will result in significant downward revisions to reimbursements for NI coding.

Contributors All authors contributed to this manuscript.

Competing interests None.

Provenance and peer review Commissioned; not externally peer reviewed.

\section{REFERENCES}

1 Silva $E$ III. Where have you gone, component coding? J Am Coll Radiol 2012;9:458-60.

2 Services CfMM. 2013 Medicare Physician Fee Schedule Final Rule [online]. https://http://www.federalregister.gov/public-inspection (accessed 21 Nov 2012).

3 Donovan WD. The resource-based relative value scale and neuroradiology: ASNR's history at the RUC. Neuroimaging Clin N Am 2012;22:421-36.

4 Moorefield JM, MacEwan DW, Sunshine JH. The radiology relative value scale: its development and implications. Radiology 1993;187:317-26.

5 Commission M. Report to the Congress: Medicare Payment Policy [online]. http://www.medpac.gov/documents/Mar06_EntireReport.pdf (accessed 20 Nov 2012).

6 Office UGA. Medicare physician payments: fees could better reflect efficiencies achieved when services are provided together [online]. http://www.gao.gov/new. items/d09647.pdf (accessed 20 Nov 2012).

7 Silva E III. New codes from a new source: the rolling five-year review. J Am Coll Radiol 2010;7:10-12.

8 Association AM. The RUC Relativity Assessment Workgroup progress report [online]. http://www.ama-assn.org/resources/doc/rbrvs/five-year-progress.pdf (accessed 19 Nov 2012).

9 Manchikanti L, Hirsch JA. Patient protection and affordable care act of 2010: a primer for neurointerventionalists. J Neurointerv Surg 2012;4:141-6.

10 American Medical Association. The RUC relativity assessment workgroup progress report. March 26, 2012

11 Silva E III. CT abdomen and pelvis: a case study in devaluation. J Am Coll Radiol 2011:8:300-1.

12 Silva E III. The search for misvalued services: why is radiology a target? J Am Coll Radiol 2012;9:7-8.

13 Kalva SP, Somarouthu B, Hirsch J. Inferior vena cava filters for the cerebrovascular patient. J Neurointerv Surg 2011;3:137-40

14 Somarouthu B, Yeddula K, Wicky $S$, et al. Long-term safety and effectiveness of inferior vena cava filters in patients with stroke. J Neurointerv Surg 2011;3:141-6.

15 Donovan WD. What is the RUC? AJNR Am J Neuroradiol 2011;32:1583-4.

16 Replace the RUC [online]. http://www.replacetheruc.org/ (accessed 20 Nov 2012).

17 Levy B. The RUC: providing valuable expertise to the Medicare program for twenty years. [online]. http://www.kaiserhealthnews.org/Columns/2011/March/032811levy. aspx (accessed 21 Nov 2012).

18 Manchikanti L, Hirsch JA, Barr RM, et al. Physician payment outlook for 2012. J Neurointerv Surg 2012;4:463-7.

19 Manchikanti L, Hirsch JA. Patient protection and affordable care act of 2010: a primer for neurointerventionalists. J Neurointerv Surg 2011;4:141-6.

20 Public Law No:111-148:H.R. 3590. Patient Protection and Affordable Care Act, 2010.

21 House Resolution 3590. Misvalued codes under the physician fee schedule. The Patient Protection and Affordable Care Act § 3134 (a) (K) (iii) (V). 